\title{
Estudio Comparativo de la Productividad de Pozos Horizontales en Yacimientos de Black Oil para Estado Seudoestable
}

\section{Comparative Study for Horizontal Wells Performance in Black Oil Reservoirs for Pseudosteady-State}

\author{
Luís Fernando Bonilla ${ }^{1}$, Germán Alberto Bonilla² y Edilberto Escalante ${ }^{3}$
}

\section{Resumen}

En este trabajo se presenta un estudio comparativo de la productividad de pozos horizontales en yacimientos de black oil para estado seudoestable, partiendo de la evaluación de las diferentes correlaciones propuestas para el cálculo de la productividad, utilizando un simulador elaborado por los autores que facilitó el cálculo del índice de productividad y validando los datos obtenidos. Para el cálculo de la productividad en el simulador numérico se describe un procedimiento comenzando desde la obtención del tiempo de inicio del estado seudoestable, la generación de la malla PEBI y finalizando con los resultados de la presión de fondo fluyendo (Pwf) necesarios para obtener el índice de productividad. La comparación y posterior análisis de los resultados generados de las correlaciones y el simulador numérico se muestran en tablas y gráficas. La correlación que más se ajusta a los resultados obtenidos en el simulador es la de Joshi, no obstante, a ésta se le realizó un ajuste y se propone una nueva correlación la cual ofrece un mejor comportamiento comparado con los resultados del simulador.

Palabras Clave: Productividad; pozos horizontales; estado seudoestable; correlaciones; simulador numérico.

\begin{abstract}
This work refers to the comparative study for horizontal wells performance in black oil reservoirs for pseudosteady-state, it initiates with evaluation of the different correlations propose for productivity calculation, using the software "OILIP" created in present study to facilitate obtaining the productivity index and validate with data realized in EXCEL. For the calculation of productivity in the numerical simulator is described a procedure that starts by finding the beginning time of the pseudosteady-state, generation of PEBI grid and finishing with the results of pressure well flowing (Pwf) necessary to obtain the productivity index. The values obtained by the correlations and the numerical simulator were showed in tables and graphs. When was compared the correlations results with those of the numerical simulator, the correlation that more adjusts to the results thrown by numerical simulator is Joshi et al, nevertheless an adjustment was realized to the above mentioned correlation which its present a better behavior compared with the results of numerical simulator.
\end{abstract}

Key Words: Performance; Horizontal well; Pseudosteady-State; Correlation; Numerical Simulator.

1 Ingeniero de Petróleos, M.Sc. Profesor Facultad de Ingeniería universidad Surcolombiana.

fbonilla@usco.edu.co.

2 Ingeniero de Petróleos Universidad Surcolombiana Facultad de Ingeniería Neiva

yermastegod@hotmail.com

3 Ingeniero de Petróleos Universidad Surcolombiana Facultad de Ingeniería.edes_8@hotmail.com 


\section{Introducción}

El progresivo aumento de la perforación de pozos horizontales ha venido ampliando la necesidad de conocer el comportamiento de la productividad de los mismos así como también los factores que influyen en el desarrollo productivo tales como las propiedades del yacimiento y la geometría de pozo con respecto al yacimiento.

Varias investigaciones para el cálculo del índice de productividad han sido presentadas por diferentes autores a través de los años para pozos horizontales, estas han sido enfocadas al estudio de las características y condiciones que se presentan en el yacimiento y su respectiva producción. Algunas de las características son los límites del yacimiento y la dependencia del tiempo que definen los diferentes cambios de presión que se pueden presentar cuando esta toca la frontera, estos son el estado estable y el seudoestable.

Este trabajo recopila las correlaciones propuestas por autores muy reconocidos en el ámbito de la investigación referente a la obtención del índice de productividad para pozos horizontales que producen en estado pseudoestable como son: Mutalik et al (1988), Babu \& Odeh (1989), Kuchuk et al(1988), Economides et al (1998) y Joshi (1991). Cada una de estas correlaciones presenta consideraciones en las diferentes variables de dependencia para el cálculo de la productividad generando que cada una difiera de la otra en su desarrollo matemático.

Estas correlaciones son las siguientes:

- Mutalik et al (1988):

$$
\begin{aligned}
& \mathrm{J}_{0}=\frac{0.007078 \mathrm{~K}_{h} h /\left(\mu_{o} \beta_{o}\right)}{\ln \left(r_{e} / r_{w}\right)-A^{\prime}+s_{C A, h}+s_{m}+s_{f}-c^{\prime}+D_{q}} \\
& J_{0}=\frac{0.007078 .\left(2 X_{e}\right) \sqrt{K_{y} K_{v}} /\left(\mu_{o} \beta_{o}\right)}{\ln \left(\sqrt{A_{1}} / r_{w}\right)+\ln C_{H}-0.75+S_{R}} \\
& J_{0}=\frac{K_{h} h /\left(70.6 \mu_{o} \beta_{o}\right)}{F+\left(\frac{h}{0.5 L}\right) \sqrt{\frac{K_{h}}{K_{v}} S_{x}}}
\end{aligned}
$$

- Economides et al (1998):

$$
J_{o}=\frac{q_{o}}{P_{e}-P w f}=\frac{\bar{K} X_{e}}{887.22 \mu_{\circ} B_{\circ}\left[P D+\frac{X_{e}}{2 \pi . L} S\right]}
$$

- Joshi (1991) :

$$
J_{0}=
$$

$$
\frac{K_{h} h}{141.2 \beta_{o} \mu_{0}\left[\ln \left[\frac{a+\sqrt{a^{2}-(L / 2)^{2}}}{L / 2}\right]+\left[\frac{I_{a n i} h}{L}\right\rceil *\left\lceil\ln \frac{I_{a n i} h}{r\left(I_{a n i}+1\right)}-0.75+S\right]\right]}
$$

Donde $\boldsymbol{J}_{o}$ es el índice de productividad (stb/(dia.psi)), $\boldsymbol{K}_{\boldsymbol{h}}$ es la permeabilidad horizontal (md), h es el espesor de la capa (ft), $\boldsymbol{\mu}_{\mathrm{o}}$ es la viscosidad del crudo (cp), Bo es el factor volumétrico del crudo 
(rb/stb), rw es el radio del pozo (ft), re es el radio externo de frontera (ft), A' es el parámetro de drenaje para área cuadrada $(=0.738)$, sCA,h es elfactor de daño según la forma de yacimiento (adimensional), sm es el factor de daño mecánico (adimensional), Sf es el factor de daño de conductividad infinita para una longitud de fractura completamente penetrada(adimensional), L es la longitud del pozo horizontal (ft), c' es una constante de conversión de forma (=1.386), Dq es el coeficiente de turbulencia (1/bopd), $\mathrm{CH}$ es el factor de forma. A1 es el área de drenaje del pozo horizontal visto en un plano vertical $(A l=2 Y e h)(\mathrm{ft})$. Los valores de $2 \mathrm{Xe}$ y $2 \mathrm{Ye}$ son las dimensiones del yacimiento (ft), el valor de SR representa el daño por penetración parcial del pozo horizontal, $\mathrm{F}$ es una función adimensional, PD es la presión adimensional. 
Los resultados de estas correlaciones son comparados con un simulador numérico que permite estimar el comportamiento del índice de productividad con mayor exactitud teniendo en cuenta los diferentes parámetros que afectan la capacidad de producción de los pozos horizontales.

\section{Metodología}

\subsection{Modelo de estudio.}

\subsubsection{Descripción del caso base.}

El caso base que se utilizó en el estudio comparativo fue tomado del documento "A COMPREHENSIVE COMPARATIVE STUDY ON ANALYTICAL PI/IPR CORRELATIONS"

(Choi et. al. 2008), en donde se realizó un estudio analítico de algunas correlaciones para el cálculo de productividad de pozos en las diferentes fases, geometría y dependencia del tiempo.

La figura 1 muestra el esquema de un pozo con un radio $r_{w}$ el cual va horizontalmente a través del yacimiento con una longitud $L$, el espesor del yacimiento es $h$ y está definido por capas impermeables o semi-permeables, el pozo se encuentra localizado a una distancia $Z_{w}$ desde el fondo del yacimiento hasta la cara del pozo.

La figura 2 muestra un pozo horizontal de longitud $\mathrm{L}$, ubicado a una distancia $X_{w}$ y $Y_{w}$ dentro del yacimiento con un área rectangular cuyas dimensiones son $2 X_{e}, 2 Y_{e}$ de espesor $\mathrm{h}$, se especifican las direcciones de las permeabilidades con el fin de agregar el concepto de anisotropía; las figuras 1 y 2 son importantes para entender cada una de las propiedades y variables que hacen parte del modelo de estudio para el cálculo del índice de productividad.
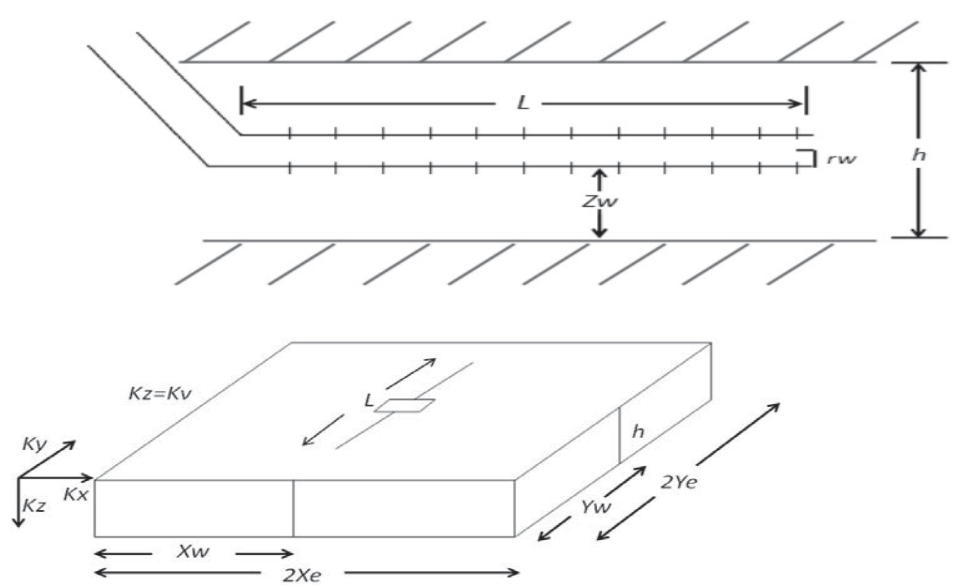

Figura 2. Esquema de un pozo horizontal localizado en un volumen rectangular de drene.

\subsubsection{Datos del caso base.}

Los datos tomados para el desarrollo del estudio comparativo son mostrados en la tabla 1. 
Revista Ingeniería y Región Vol: 6 No. 1

Tabla 1. Datos del caso base.

\begin{tabular}{|c|c|c|}
\hline Variables & Unidad & Descripción \\
\hline $\mathrm{P}_{\mathrm{e}} 3500$ & Psi & Presión de frontera \\
\hline $\mathrm{P}_{\mathrm{r}} 2500$ & Psi & Presión promedio de yacimiento \\
\hline K 108 & $\mathrm{Md}$ & Permeabilidad promedio \\
\hline $\mathrm{K}_{\mathrm{v}} 80$ & Md & Permeabilidad vertical \\
\hline $\mathrm{K}_{\mathrm{h}} 125$ & Md & Permeabilidad horizontal \\
\hline $\mathrm{K}_{\mathrm{x}} 168$ & Md & Permeabilidad en $X$ \\
\hline $\mathrm{K}_{\mathrm{y}} 93$ & Md & Permeabilidad en Y \\
\hline $\mathrm{H} 70$ & $\mathrm{Ft}$ & Espesor de la capa \\
\hline $\mathrm{r}_{\mathrm{e}} 2980$ & $\mathrm{Ft}$ & Radio externo de frontera \\
\hline$X_{e} 2640$ & $\mathrm{Ft}$ & Longitud media del yacimiento en dirección paralela a la cara del pozo \\
\hline Ye 2640 & $\mathrm{Ft}$ & Longitud media del yacimiento en dirección perpendicular a la cara del pozo \\
\hline$\beta_{0} 1.1$ & $\mathrm{rb} / \mathrm{stb}$ & Factor volumétrico del crudo \\
\hline$\mu_{\mathrm{o}} 1.7$ & $\mathrm{Cp}$ & Viscosidad del crudo \\
\hline$\rho_{0} 52$ & $\mathrm{lb} / \mathrm{ft} 3$ & Densidad del crudo \\
\hline$q_{0} 500$ & $\mathrm{stb} / \mathrm{d}$ & Caudal del crudo \\
\hline $\mathrm{r}_{\mathrm{w}} 0.328$ & $\mathrm{Ft}$ & Radio del pozo \\
\hline L 2000 & $\mathrm{Ft}$ & Longitud del pozo horizontal \\
\hline$X_{w} 2640$ & $\mathrm{Ft}$ & Distancia desde la frontera perpendicular al punto medio de la cara del pozo \\
\hline $\mathrm{Y}_{\mathrm{w}} 2640$ & $\mathrm{Ft}$ & Distancia desde la frontera paralela al centro de la cara del pozo \\
\hline $\mathrm{Z}_{\mathrm{w}} 35$ & $\mathrm{Ft}$ & Distancia desde el tope o base de la capa hasta el centro de la cara del pozo \\
\hline S 0 & 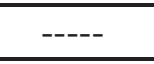 & Factor de daño \\
\hline $\mathrm{A}^{\prime} .738$ & ----- & Parámetro de drenaje para área cuadrada \\
\hline $\mathrm{C}^{\prime} 1.386$ & $\begin{array}{c}---- \\
\end{array}$ & Constante de conversión de forma \\
\hline $\mathrm{D}_{\mathrm{q}} 0$ & 1/bopd & Coeficiente de turbulencia \\
\hline
\end{tabular}

\subsection{Modificación de variables del caso base para el estudio del comportamiento de la productividad.}

\subsubsection{Modificación de las propiedades del yacimiento.}

En la tabla 2 se muestran las variables que se estudiaron, lo mismo que sus rangos de valores para ver su influencia en el comportamiento de la productividad. 
Tabla 2. Rango de propiedades estudiadas.

\begin{tabular}{|l|c|c|c|c|c|c|c|c|c|c|c|}
\hline \multicolumn{1}{|c|}{ Propiedad } & \multicolumn{9}{c|}{ Valor } \\
\hline $\begin{array}{l}\text { Factor volumétrico del } \\
\text { crudo (rb/stb) }\end{array}$ & 1 & 1.1 & 1.2 & 1.3 & 1.4 & 1.5 & 1.6 & 1.7 & 1.8 & & \\
\hline Viscosidad del crudo (cp) & 0.5 & 1 & 1.7 & 2 & 3 & 5 & 8 & 10 & 12 & & \\
\hline Permeabilidad promedio (md) & 10.5 & 42 & 73 & 94 & 108 & 120 & 151 & 182 & 202 & & \\
\hline Relación de permeabilidad (K/K $\left./ \mathrm{K}_{v}\right)$ & 0.25 & 0.5 & 1 & 2 & 4 & & & & & & \\
\hline Radio del pozo (ft) & 0.1 & 0.2 & 0.3 & 0.328 & 0.4 & 0.5 & 0.6 & 0.7 & 0.8 & 0.9 & 1 \\
\hline Radio externo de frontera (ft) & 1693 & 2257 & 2822 & 2980 & 3386 & 3950 & 4515 & 5079 & & & \\
\hline Espesor de la capa (ft) & 25 & 50 & 70 & 100 & 150 & 200 & 250 & 300 & 350 & 400 & \\
\hline
\end{tabular}

Para variar algunas propiedades es necesario tener en cuenta cambios en otras:

- $\quad$ Permeabilidad promedio: La K promedio se calcula con $\bar{K}=\sqrt[3]{K_{x} \cdot K_{y} \cdot K_{z}}$ donde $K z=K v$, Por tal motivo se debe guardar proporción en la variación de todas las permeabilidades de diferentes direcciones.

- $\quad$ Relación de permeabilidad vertical y horizontal: Como el objetivo es analizar la relación entre permeabilidad horizontal y vertical, $K x$ y $K y$ deben ser iguales ya que

$$
K_{h} \quad=\sqrt[2]{K_{x} \cdot K_{y}}
$$

- Radio externo de frontera: El radio externo de frontera es afectado directamente por el tamaño del yacimiento por este motivo también se deben cambiar $X e$, Ye como también $X w$ y $Y w$, ya que el pozo debe estar centrado.

- Espesor de la capa: Al modificar el espesor de la capa se debe cambiar la ubicación del pozo en dirección vertical $Z w$ para asegurarse que se encuentre centrado.

\subsubsection{Modificación de los parámetros operacionales.}

$\mathrm{Al}$ igual que las propiedades del yacimiento, se modificaron algunos parámetros geométricos (ver tabla 3) con el fin de observar su influencia.

Tabla 3. Parámetros operacionales.

\begin{tabular}{|c|c|c|c|c|c|c|c|c|c|c|c|}
\hline Parámetro & \multicolumn{11}{|c|}{ Valor } \\
\hline $\begin{array}{l}\text { Longitud del } \\
\text { pozo horizontal }\end{array}$ & 300 & 600 & 900 & 1200 & 1500 & 1800 & 2000 & 2100 & 2400 & 2700 & 3000 \\
\hline Excentricidad Z $(\mathrm{ft})$ & 17.5 & 35 & 52.5 & 61.3 & & & & & & & \\
\hline \multicolumn{12}{|c|}{ Ubicación del pozo en el yacimiento } \\
\hline \multicolumn{12}{|c|}{ Centrado } \\
\hline \multicolumn{12}{|c|}{ Descentrado a la izquierda } \\
\hline \multicolumn{12}{|c|}{ Descentrado a la derecha } \\
\hline \multicolumn{12}{|c|}{ Descentrado en esquina inferior izquierda } \\
\hline Descentrado en esquin & erior & erech & & & & & & & & & \\
\hline
\end{tabular}

Como la longitud del pozo horizontal es uno de los parámetros operacionales más importantes, se realizó un estudio de la variación de longitud para cuatro diferentes radios externos de frontera. Para la excentricidad se tomaron los datos estándar del modelo, teniendo en cuenta el espesor $h=70 \mathrm{ft}$. Cada una de estas variables fue modificada tanto en las diferentes correlaciones utilizando el simulador desarrollado para el cálculo de la productividad como también en el simulador numérico comercial siguiendo un procedimiento adecuado. 


\section{3 Modificación de la correlación de Joshi}

Para el ajuste de la correlación de Joshi se buscaron dos factores $\boldsymbol{Y}$ y $\boldsymbol{W}$ que multiplicaran ambos flujos "el horizontal y el vertical" que están representados en el denominador de la ecuación. Los valores de estos factores se muestran en la tabla 4.

$J_{0}$

$$
K h * h
$$

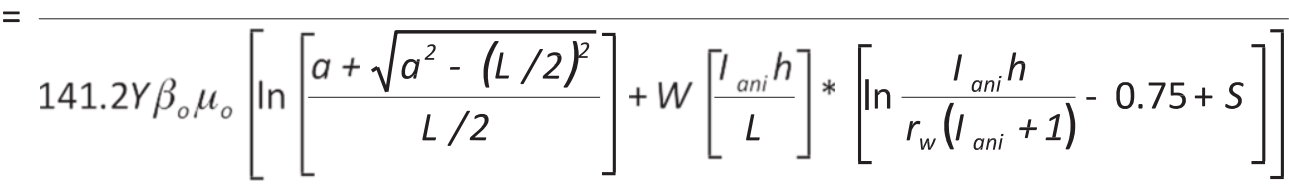

Se realizaron diversos cálculos para hallar los valores apropiados para Y y W tales que el valor del índice de productividad sea igual resultado del simulador; estos valores fueron hallados utilizando métodos numéricos y el programa Solver que es una herramienta de aplicación de Microsoft Excl. Los resultados de estos cálculos son mostrados en la tabla 4.

\begin{tabular}{|c|c|c|c|}
\hline Parámetro & IP [bbl/(psi*dia)] & $\mathbf{Y}$ & $\mathbf{W}$ \\
\hline$\beta_{0}=1 \mathrm{rb} / \mathrm{stb}$ & 14.368 & 1.22315703 & 1.2452625 \\
\hline$\beta_{0}=1,4 \mathrm{rb} / \mathrm{stb}$ & 9.580 & 1.30466874 & 1.26613307 \\
\hline$\beta_{0}=1,8 \mathrm{rb} / \mathrm{stb}$ & 7.980 & 1.22373809 & 1.24432371 \\
\hline $\mathrm{h}=50 \mathrm{ft}$ & 9.64 & 1.22007675 & 1.24335688 \\
\hline $\mathrm{h}=150 \mathrm{ft}$ & 24.60 & 1.22990029 & 1.24557687 \\
\hline $\mathrm{h}=350 \mathrm{ft}$ & 42.50 & 1.24480147 & 1.24811813 \\
\hline $\mathrm{r}_{\mathrm{e}}=2257 \mathrm{ft} ; \mathrm{L}=600 \mathrm{ft}$ & 7.600 & 1.27600417 & 1.25315755 \\
\hline$r=2257 \mathrm{ft} ; \mathrm{L}=2000 \mathrm{ft}$ & 11.840 & 1.52919158 & 1.32940296 \\
\hline $\mathrm{r}_{\mathrm{e}}=2257 \mathrm{ft} ; \mathrm{L}=2700 \mathrm{ft}$ & 13.740 & 1.61314849 & 1.3637344 \\
\hline $\mathrm{r}_{\mathrm{e}}=2980 \mathrm{ft} ; \mathrm{L}=600 \mathrm{ft}$ & 7.473 & 1.19479182 & 1.2906482 \\
\hline $\mathrm{r}_{\mathrm{e}}=2980 \mathrm{ft} ; \mathrm{L}=2000 \mathrm{ft}$ & 13.062 & 1.21078363 & 1.294534 \\
\hline $\mathrm{r}_{\mathrm{e}}=2980 \mathrm{ft} ; \mathrm{L}=2700 \mathrm{ft}$ & 15.425 & 1.22792793 & 1.29947845 \\
\hline $\mathrm{r}_{\mathrm{e}}=3950 \mathrm{ft} ; \mathrm{L}=600 \mathrm{ft}$ & 6.420 & 1.29010526 & 1.30777409 \\
\hline $\mathrm{r}_{\mathrm{e}}=3950 \mathrm{ft} ; \mathrm{L}=2000 \mathrm{ft}$ & 9.860 & 1.40306037 & 1.33388933 \\
\hline $\mathrm{r}=3950 \mathrm{ft} ; \mathrm{L}=2700 \mathrm{ft}$ & 10.74 & 1.49828289 & 1.36113873 \\
\hline $\mathrm{r}=5079 \mathrm{ft} ; \mathrm{L}=600 \mathrm{ft}$ & 6.270 & 1.24078723 & 1.32437622 \\
\hline $\mathrm{r}=5079 \mathrm{ft} ; \mathrm{L}=2000 \mathrm{ft}$ & 9.840 & 1.27265951 & 1.33075865 \\
\hline $\mathrm{r}=5079 \mathrm{ft} ; \mathrm{L}=2700 \mathrm{ft}$ & 11.17 & 1.28928706 & 1.33464712 \\
\hline$\mu_{\mathrm{O}}=1 \mathrm{cp}$ & 20.372 & 1.29311149 & 1.39829684 \\
\hline$\mu_{\mathrm{O}}=5 \mathrm{cp}$ & 4.251 & 1.2423546 & 1.38580118 \\
\hline$\mu_{\mathrm{O}}=10 \mathrm{cp}$ & 2.233 & 1.18562826 & 1.37228324 \\
\hline \multicolumn{2}{|c|}{ Valores de $\mathrm{Y}$ y $\mathrm{W}$ promediados } & 1.29128053 & 1.30728191 \\
\hline
\end{tabular}

Tabla 4. Cálculo de los factores Y y W 
Reemplazando los valores la ecuación modificada de Joshi queda de la siguiente manera:

$$
J_{0}=\frac{K h^{*} h}{182.329 \beta_{0} \mu_{0}\left[\ln \left[\frac{a+\sqrt{a^{2}-(L / 2)^{2}}}{L / 2}\right]+1.3073\left[\frac{I_{a n i} h}{L}\right] *\left[\ln \frac{I_{a n i} h}{r w\left(I_{a n i}+1\right)}-0.75+S\right]\right]}
$$

\section{Resultados}

Los resultados obtenidos de las correlaciones y la simulación numérica son presentados a continuación:

\subsection{Modificación de las propiedades del yacimiento.}

Las figuras de la 3 a la 9 muestran el comportamiento de la productividad con respecto a los diferentes rangos aplicados de cada variable, con cada una de las correlaciones y el simulador numérico. En ellas se puede observar el orden de proximidad de los resultados obtenidos de las correlaciones con el simulador y se nota que la correlación propuesta por Joshi es la que más se acerca y contrariamente la de Economides et al. es la que más difiere.

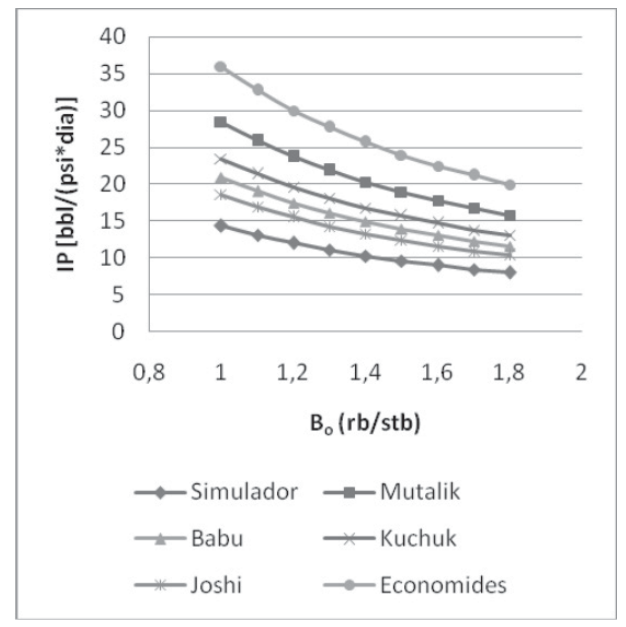

Figura 3. Comportamiento de la productividad del pozo variando el factor volumétrico del crudo.

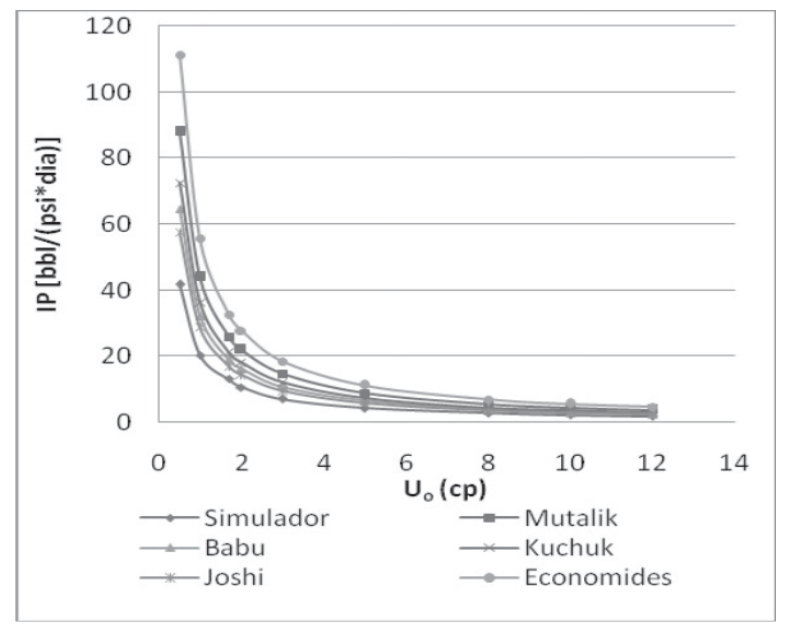

Figura 4. Comportamiento de la productividad del pozo variando la viscosidad del crudo. 
Revista Ingeniería y Región Vol: 6 No. 1

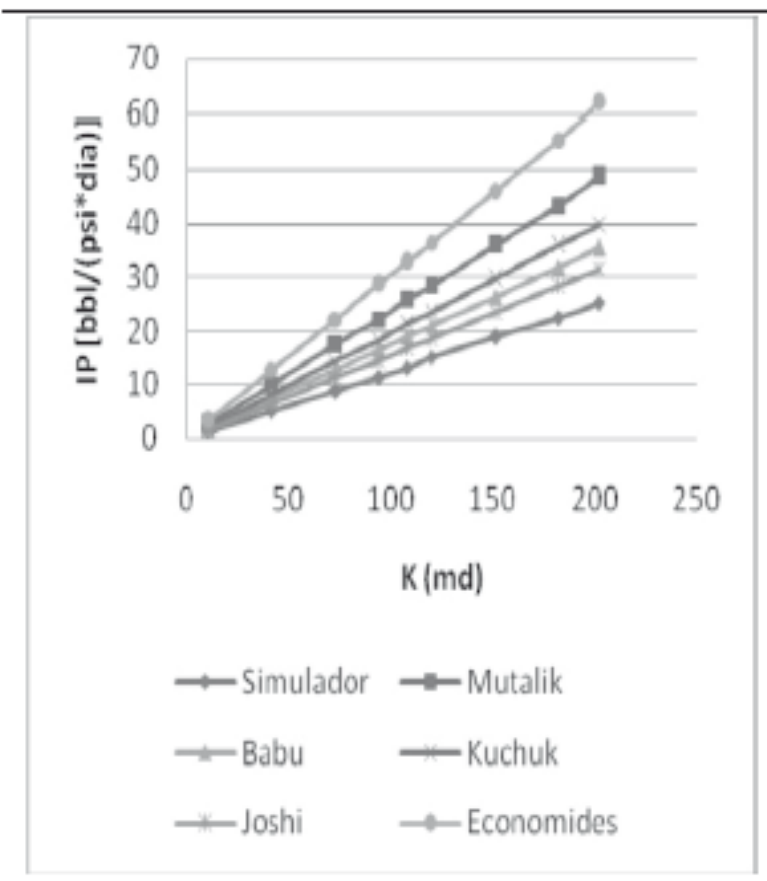

Figura 5. Comportamiento de la productividad del pozo variando la permeabilidad promedio del crudo.

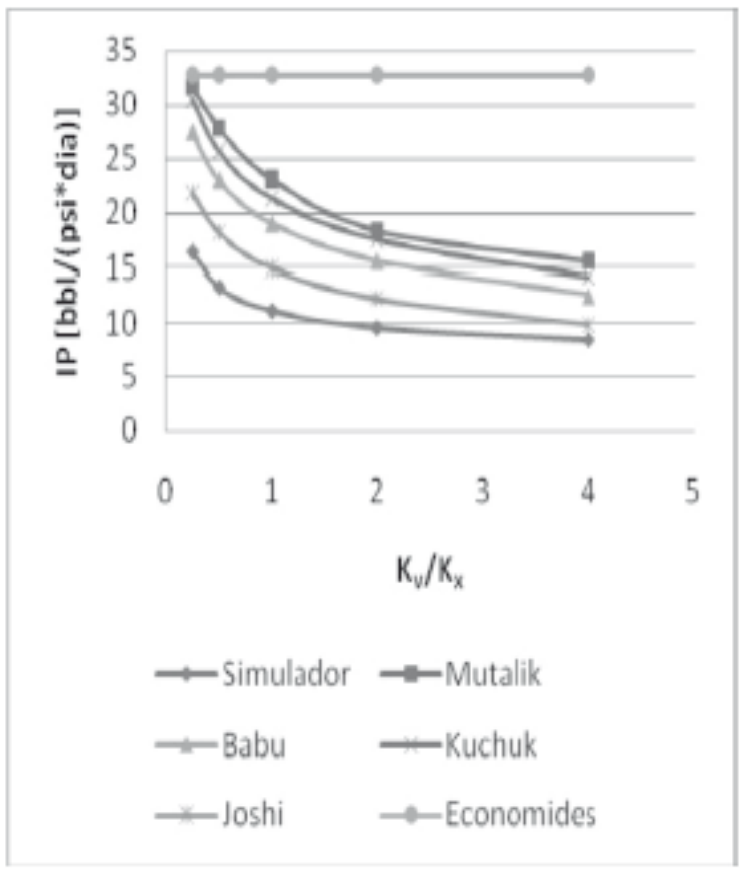

Figura 6. Comportamiento de la productividad del pozo variando la relación de permeabilidad verticalhorizontal.

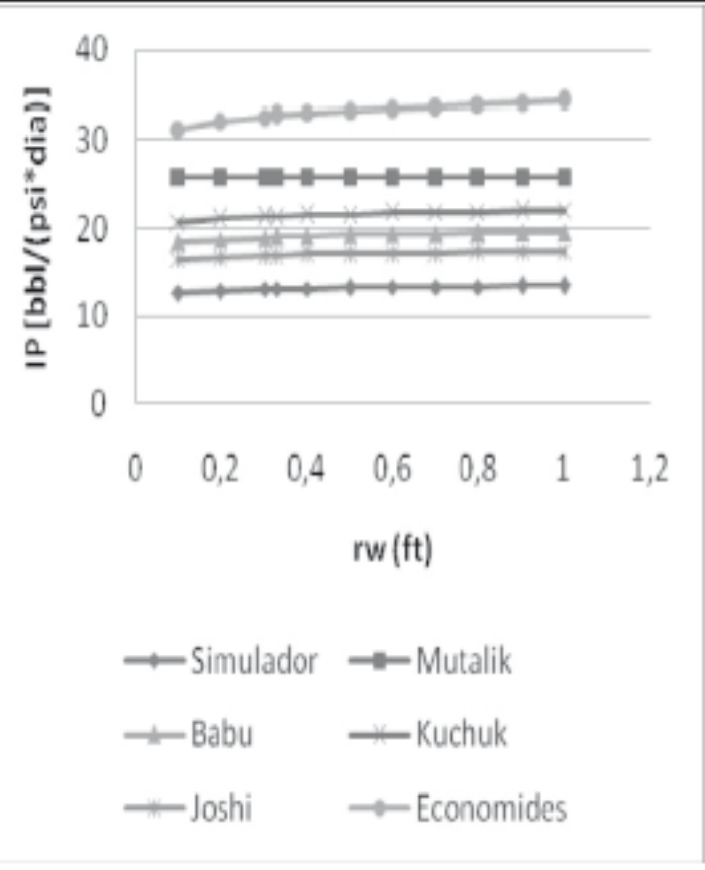

Figura 7. Comportamiento de la productividad del pozo variando el radio del pozo.

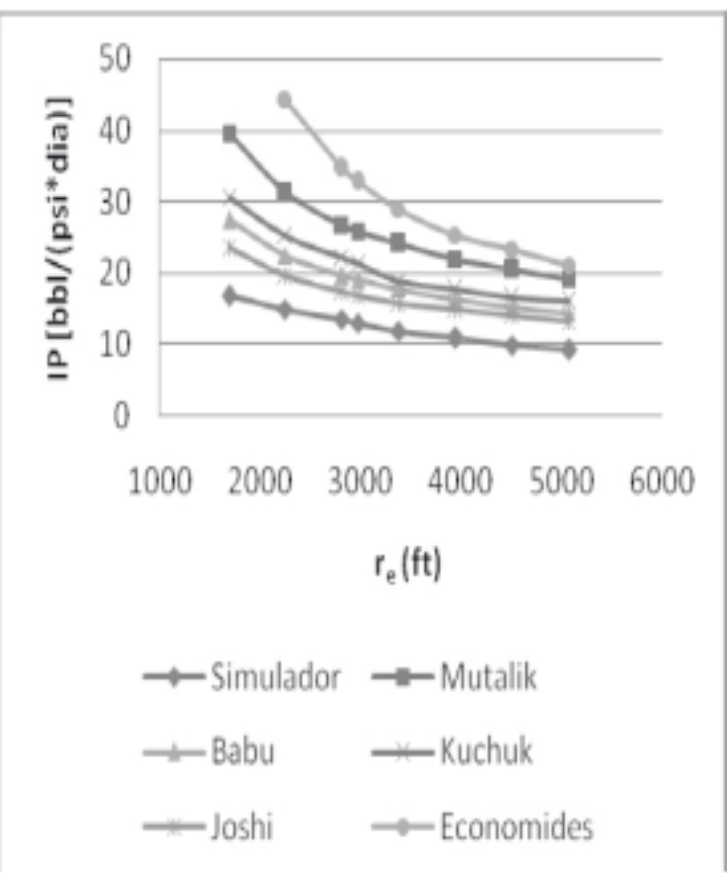

Figura 8. Comportamiento de la productividad del pozo variando el radio externo de frontera. 


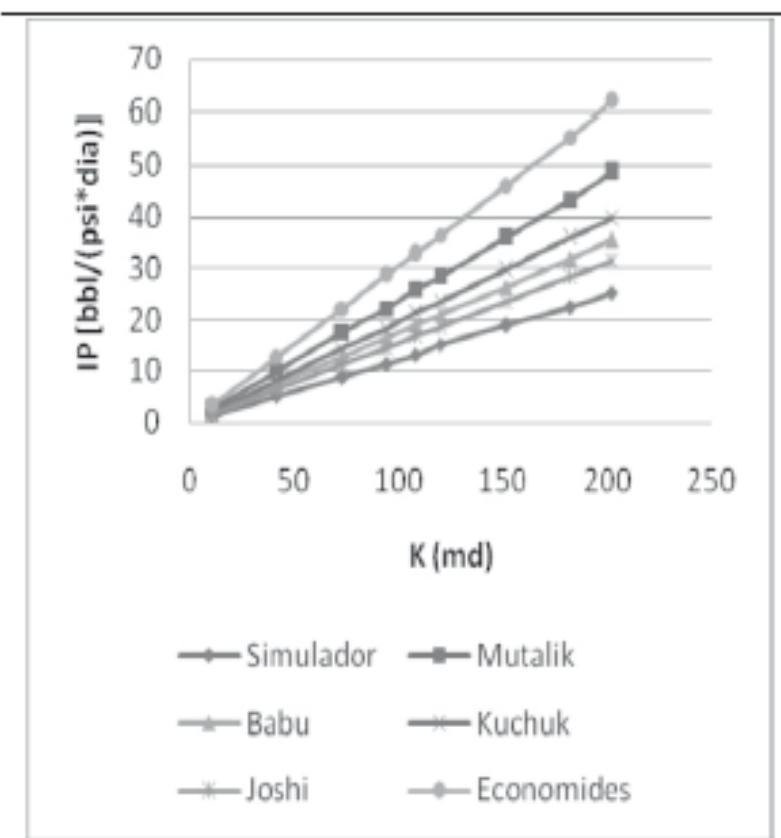

Figura 5. Comportamiento de la productividad del pozo variando la permeabilidad promedio del crudo.

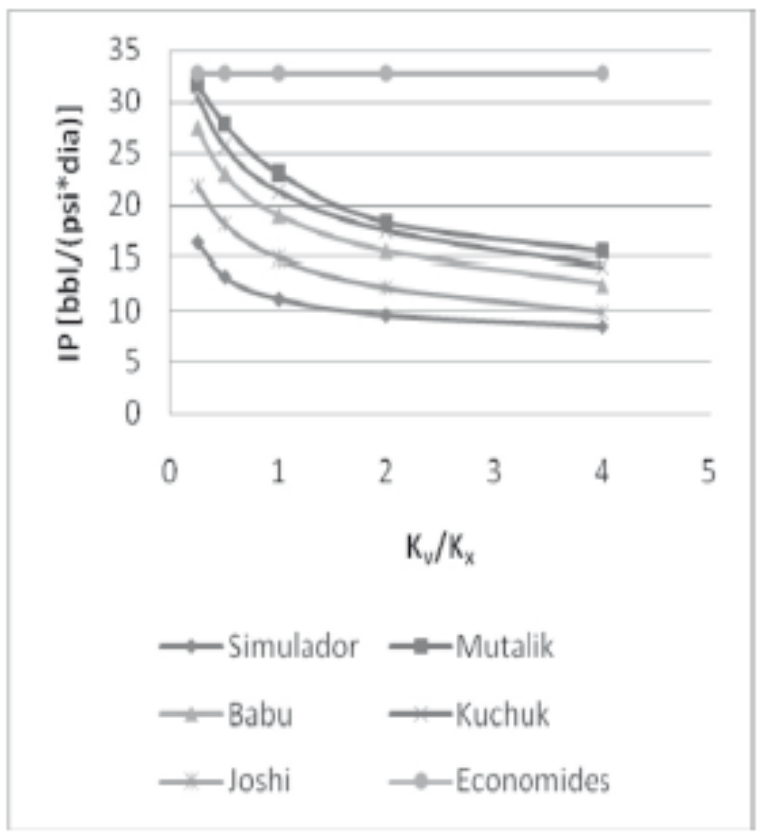

Figura 6. Comportamiento de la productividad del pozo variando la relación de permeabilidad verticalhorizontal.

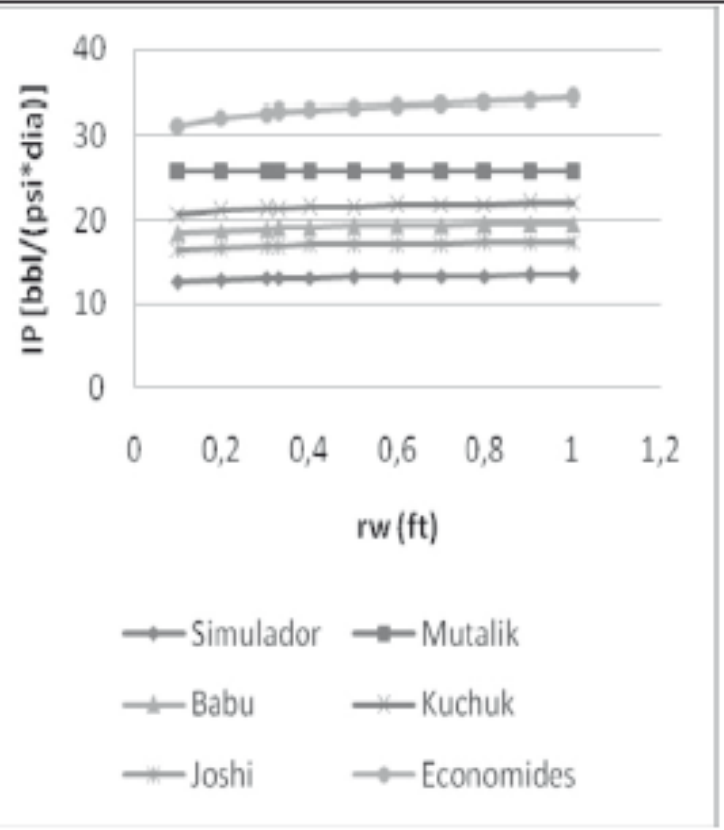

Figura 7. Comportamiento de la productividad del pozo variando el radio del pozo.

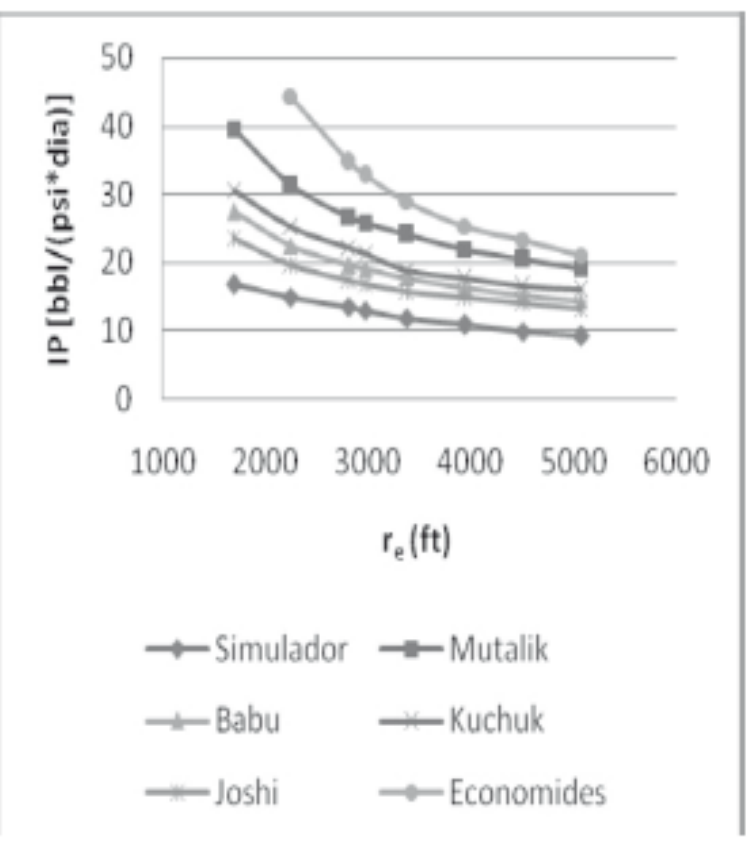

Figura 8. Comportamiento de la productividad del pozo variando el radio externo de frontera. 


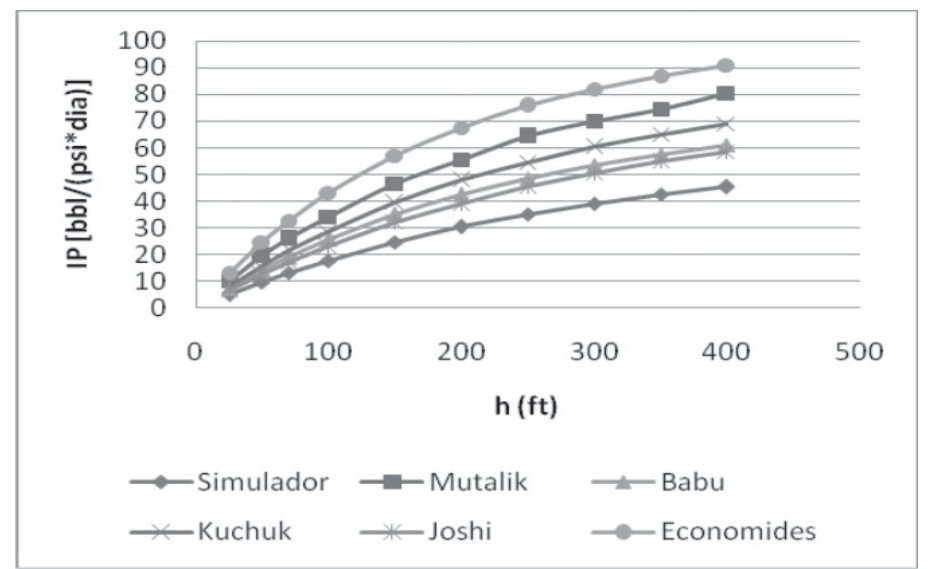

Figura 9. Comportamiento de la productividad del pozo variando el espesor de la capa.

\subsection{Modificación de los parámetros operacionales}

Las figuras de la 9 a la 11 muestran el comportamiento de la productividad con respecto a los diferentes rangos aplicados de cada variable del parámetro operacional para cada una de las correlaciones y el simulador numérico.

Se observa en la figura 9 que las correlaciones de Kuchuk et al, Mutalik et al y Economides et al. presentaron limitaciones en cuanto al comienzo de los rangos propuestos para las longitudes. El orden de proximidad de los resultados de las correlaciones con el simulador son respectivamente: Joshi, Babu y Odeh, Kuchuk et al, Mutalik et al y Economides et al.

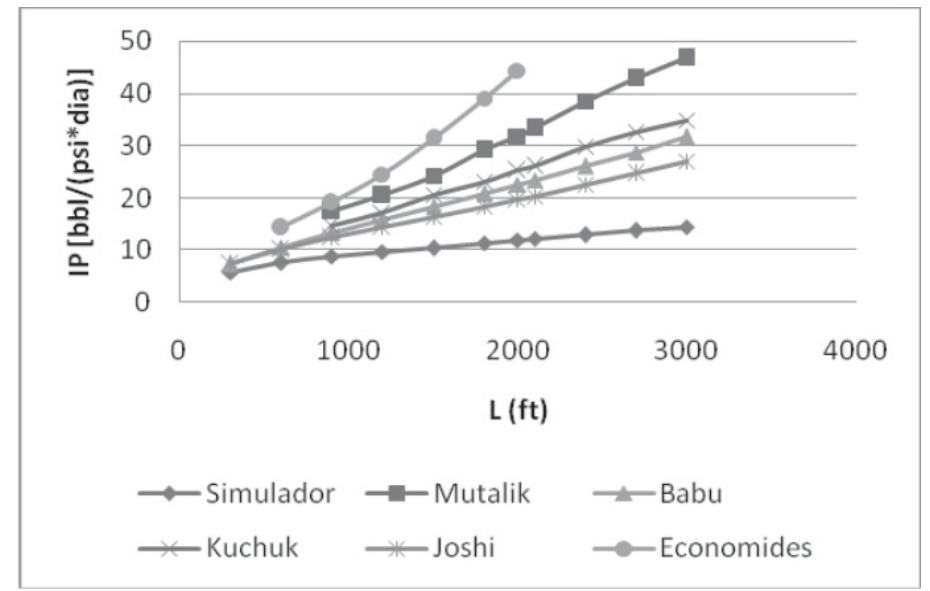

Figura 9. Comportamiento de la productividad del pozo variando la longitud horizontal para

$$
r_{e}=2980 \mathrm{ft} .
$$


En la figura 10 se observa la sensibilidad que la productividad tiene en función de la excentricidad del pozo.

Entre más centrado esté el pozo en el estrato productor mejor es el índice de productividad.

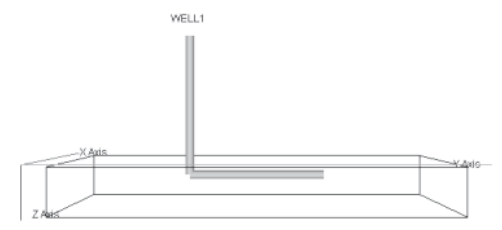

$\mathrm{Z}_{\mathrm{w}}=52.5 \mathrm{ft}$

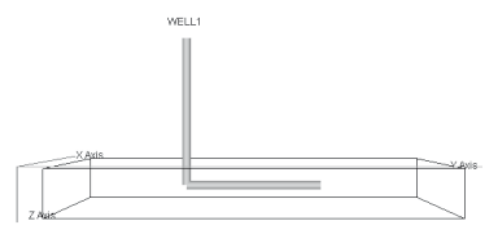

$\mathrm{Z}_{\mathrm{w}}=52.5 \mathrm{ft}$ (centrado)

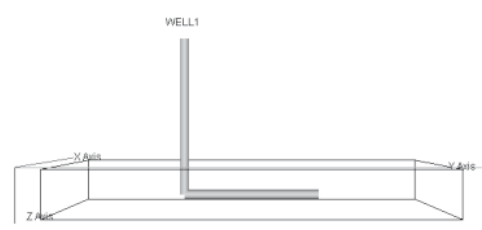

$\mathrm{Z}_{\mathrm{w}}=52.5 \mathrm{ft}$

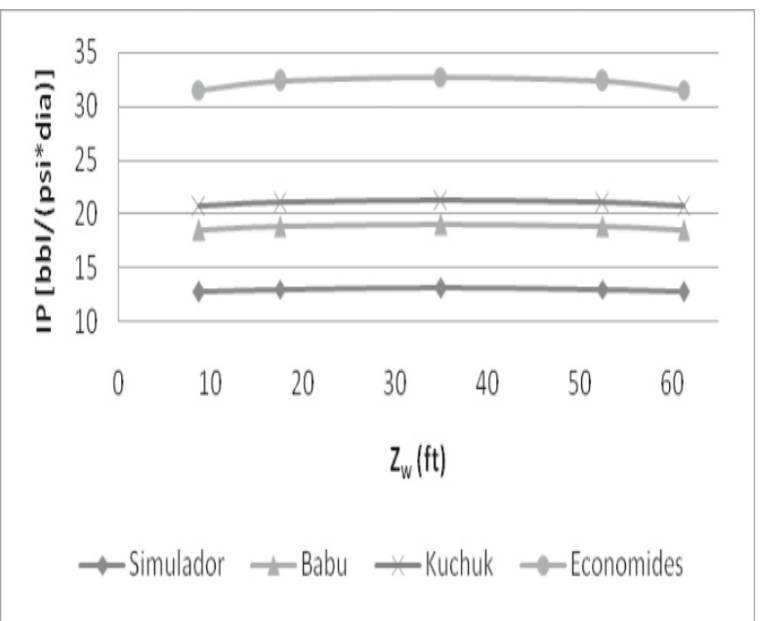

Figura 10. Comportamiento de la productividad del pozo variando la excentricidad en el eje vertical.

La figura 11 muestra las tres ubicaciones del pozo con respecto a las fronteras laterales del yacimiento y la tabla 5 muestra la sensibilidad que tiene el índice de productividad de acuerdo a la ubicación del pozo. Se puede establecer que entre más centrado esté el pozo mejor es su índice de productividad.

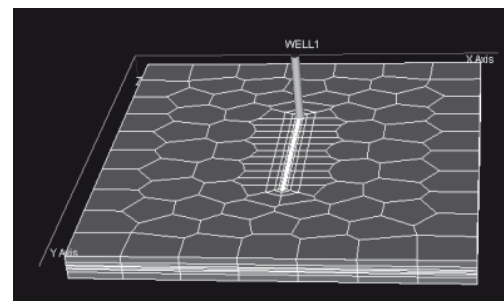

Centrado

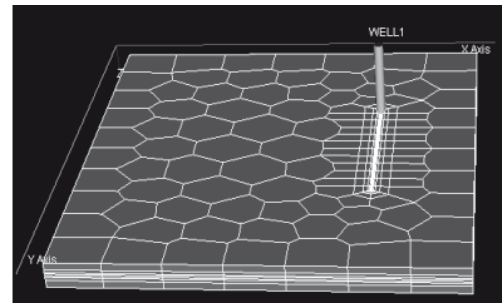

Descentrado a la derecha

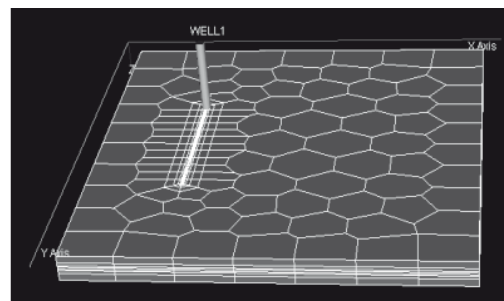

Descentrado a la izquierda

Figura 11. Esquemas de ubicación del pozo horizontal vistas en el simulador. 
Tabla 5. Comportamiento de la productividad del pozo variando la ubicación del pozo en el yacimiento.

\begin{tabular}{|c|c|c|c|c|c|c|}
\hline \multirow{2}{*}{ Ubicación } & \multicolumn{7}{|c|}{ IP [bbl/(psi*dia)] } \\
\cline { 2 - 7 } & Simulador & Mutalik & Babu & Kuchuk & Joshi & Economides \\
\hline Centrado & 13.06 & 25.85 & 18.98 & 21.32 & 16.89 & 32.7 \\
\hline Descentrado a la izquierda & 11.73 & 25.85 & 15.85 & 17.18 & 16.89 & 32.7 \\
\hline Descentrado a la derecha & 11.73 & 25.85 & 15.85 & 17.18 & 16.89 & 32.7 \\
\hline $\begin{array}{c}\text { Des. esquina } \\
\text { inferior }\end{array}$ & 8.94 & 25.85 & 9.96 & 11.35 & 16.89 & 32.7 \\
\hline $\begin{array}{c}\text { Des. esquina } \\
\text { superior }\end{array}$ & 8.94 & 25.85 & 9.96 & 11.35 & 16.89 & 32.7 \\
\hline
\end{tabular}

3.3 Comparación de la correlación de Joshi y el simulador comercial con la correlación modificada de Joshi.

Los resultados obtenidos por la correlación de Joshi y el simulador son comparados con una nueva correlación desarrollada a partir de un ajuste que se le realizó a la propuesta por Joshi. Los resultados del índice de productividad generados por la correlación modificada de Joshi presentaron un mejor acercamiento a los resultados presentados por el simulador numérico para todos los casos estudiados, con un porcentaje de error que varía de 1 a $10 \%$.

La figura 12 muestra la comparación entre Joshi, Joshi modificado y el simulador variando el espesor de la capa.

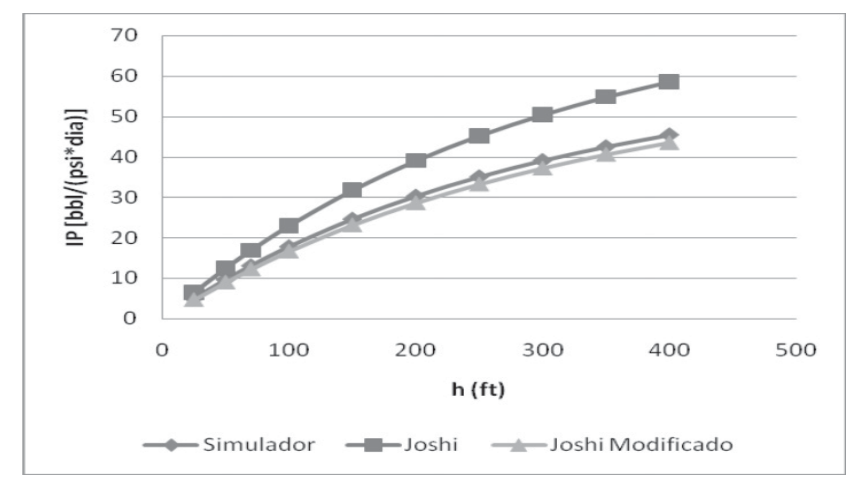

Figura. 12. Comportamiento de la productividad del pozo variando el espesor de la capa.

\section{Conclusiones}

Se compararon correlaciones para el cálculo del índice de productividad en pozos horizontales para estado seudoestable con un simulador numérico, observándose grandes variaciones con respecto al simulador.

Entre las correlaciones comparadas, la propuesta por Joshi es la que más se acerca al comportamiento mostrado por el simulador numérico.

Se determinaron los límites de aplicación de las correlaciones utilizadas para el cálculo de la productividad. 
Se creó un software llamado "OILIP" para el cálculo y modificación de las variables influyentes en el índice de productividad para cada una de las correcciones.

Se propone unos factores para ajustar la correlación presentada por Joshi, obteniendo resultados más cercanos al comportamiento expuesto por el simulador numérico.

\section{Referencias Bibliográficas}

1. Babu, D.K. and Odeh, A.S. 1989. Productivity of a Horizontal Well. Paper SPE 417421. SPE Reservoir Egineering.

2. Choi, S.K., Ouyang, L.B. and Huang, W.S. 2008. A Comprehensive Comparative Study on Analytical PI/IPR Correlations. Paper SPE 116580. SPE Annual Technical Conference and Exhibition Held. Denver, Colorado, U.S.A

3. Economides, M.J. and Retnanto, A. 1998. Inflow Performance Relationships of Horizontal and Multibranched Wells. Paper SPE 49054. SPE Annual Technical Conference and Exhibition Held. New Orleands, U.S.A.

4. Joshi, S. D. 1991. Horizontal Well Technology. Tulsa, OK, U.S.A: Pennwell Books.

5. Kuchuk, F.J., Goode, P.A., Brice, B.W., Sherrard, D.W., and Thambynayagam, M. 1988. Pressure Transient Analysis and Inflow Performance for Horizontal Wells. Paper SPE 18300. SPE 63rd Annual Technical conference and exhibition. Houston, U.S.A.

6. Mutalik, P.N., Godbole, S.P., and Joshi, S.D. 1988. Effect of Drainage Area Shapes on Horizontal Well

Productivity. Paper SPE 18301. SPE 63rd Annual Technical conference and exhibition. Houston, U.S.A.

7. Suk Kyoon Choi, Liang-Biao Ouyang Y Wann-Sheng (Bill) Huang. 2008. A Comprehensive Comparative Study on Analytical PI/IPR Correlations. Paper SPE 116580. Annual Technical conference and exhibition Held. Denver, Colorado, U.S.A. 